\title{
Schizophrenia and the Scaffolded Self
}

\author{
Joel Krueger ${ }^{1} \mathbb{D}$
}

Published online: 8 March 2018

(c) The Author(s) 2018. This article is an open access publication

\begin{abstract}
A family of recent externalist approaches in philosophy of mind argues that our psychological capacities are synchronically and diachronically "scaffolded" by external (i.e., beyond-the-brain) resources. I consider how these "scaffolded" approaches might inform debates in phenomenological psychopathology. I first introduce the idea of "affective scaffolding" and make some taxonomic distinctions. Next, I use schizophrenia as a case study to argue—along with others in phenomenological psychopathology — that schizophrenia is fundamentally a self-disturbance. However, I offer a subtle reconfiguration of these approaches. I argue that schizophrenia is not simply a disruption of ipseity or minimal self-consciousness but rather a disruption of the scaffolded self, established and regulated via its ongoing engagement with the world and others. I conclude by considering how this scaffolded framework indicates the need to consider new forms of intervention and treatment.
\end{abstract}

Keywords Externalism · Embodiment · Scaffolded cognition · Emotions · Phenomenological psychopathology · Schizophrenia $\cdot$ Self-disorder

\section{Introduction}

A family of recent externalist approaches in philosophy of mind argues that minds are more than brains. For these approaches, our psychological capacities are realized not just by our brains but also by our bodies, as well as the complex ways these bodies interact with their material and social environments. Some working in this area have recently developed externalist approaches to affect. They argue that moods and emotions, for example, can be synchronically and diachronically "scaffolded" by external (i.e., beyondthe-brain) resources that actively regulate the qualitative and temporal character of the state in question. From this perspective, the physical basis of these states may not be restricted to the individual but instead encompass external resources-e.g., artefacts, technologies, and other peoplewith which she is closely connected.

Despite much recent interest in this topic, it has not yet found its way to philosophy of psychiatry in a substantive way. ${ }^{1}$ This is surprising. Since disturbances of affectivity

Joel Krueger

j.krueger@exeter.ac.uk

1 Sociology, Philosophy, and Anthropology, University of Exeter, Amory Building, Rennes Drive, Exeter EX4 4RJ, UK figure so prominently in a wide variety of mental disorders, the topic seems like a fruitful place to bridge externalist paradigms and psychiatry. In what follows, I attempt to build such a bridge. I have two main objectives: first, I introduce the idea of "affective scaffolding". I distinguish three forms of affective scaffolding and support this taxonomy by appealing to different sources of empirical work. Second, I put the idea of affective scaffolding to work in the context of psychopathology. Using schizophrenia as a case study, I argue-along with others in phenomenological psychopathology - that schizophrenia is fundamentally a self-disturbance. However, I offer a subtle reconfiguration of these approaches. I argue that schizophrenia is not simply a disruption of ipseity or minimal self-consciousness but rather a disruption of the scaffolded self, established and regulated via its ongoing engagement with the world and others. I conclude that this way of thinking about the scaffolded self is potentially transformative both for our theoretical as well as practical understanding of the causes and character of schizophrenic experience, insofar as it suggests the need to consider new forms of intervention and treatment.

\footnotetext{
${ }^{1}$ Cooper (2017), Drayson (2009), Hoffman (2016), and Sprevak (2011) all provide helpful discussions of how externalist approaches to cognition might inform thinking about mental disorders. However, only Hoffman mentions emotions - and while suggestive, this part of her discussion is quite brief.
} 


\section{Externalising Affect}

Externalist approaches to cognition argue that cognitive processes like believing and remembering depend upon resources beyond the head. The various tools and technologies we use to support these processes play such a crucial role in "scaffolding" (i.e., setting up, driving, and regulating) them, we ought to view these external resources as part of the cognitive process itself (Clark 2008) —or at least as contributing environmental support essential for maintaining the character and functional integrity of the process in question (Sterelny 2010). ${ }^{2}$ A prominent trend in recent externalist debates considers the possibility that affective states like moods and emotions might be similarly scaffolded (Carter et al. 2016; Colombetti and Roberts 2015; Greenwood 2013; Krueger 2014b; Krueger and Szanto 2016; Slaby 2014; Stephan et al. 2014). Call this the scaffolded affectivity thesis (SA). One way to motivate SA is by investigating the different strategies we use to regulate affect. Traditionally, affect regulation has been seen as a process that occurs almost entirely inside people's heads (e.g., Gross 2015). But this internalist supposition ignores emergent qualities of affect regulation that only arise via an individual's ongoing engagement with features of their extra-neural body, as well as their material and social environment (Koole and Veenstra 2015).

Everyday life reveals many cases where we "offload" part the regulatory process onto the world; it loops through different parts of our environment before coming back to us, shaping our experience and behavior. Importantly, these processes constitutively depend on the input of external resources for their format and dynamics. For example, we play or listen to music, drink wine, light candles, wear specific items of clothing, or seek familiar spaces and friends to evoke and regulate specific affective experiences that reflect idiosyncratic details of the resource(s) involved (Colombetti and Krueger 2015; Maiese 2016). Listening to a favorite

\footnotetext{
${ }^{2}$ Although I am sympathetic to extended approaches to cognition generally, I will here follow Sterelny and use "scaffolding" to refer to the many ways resources beyond brain and body act as "fuels for cognition" (Sterelny 2010, p. 473). For Sterelny, discussions of extended cognition (e.g., Clark 2008) focus excessively on single agents interacting with informational resources (notebooks, smartphones, etc.) to enhance their cognitive capacities-an individualistic focus that "obscures rather than highlights" the many ways we engineer our environments to augment intelligent action on both an individual and collective level (Sterelny 2010, p. 473). A scaffolded perspective is a more general-and thus for Sterelny, more powerful-framework than a (merely) extended approach. Crucially, it affirms the importance of including resources beyond brain and body when explaining the causes and character of cognition. But it does so without becoming mired in unhelpful "causal-constitution" debates (e.g., Adams and Aizawa 2008). For more on this latter point, see Colombetti and Krueger (2015, pp. 1157-1160).
}

song, for example, will regulate our affective state in a way qualitatively different than will dressing up in a favorite shirt, decluttering our office to alleviate stress, or commiserating with a close friend.

Not every environmental interaction supports SA. It's unclear that simply coming home and lighting candles to relax after a stressful day scaffolds my affective state in the relevant sense. The causal relation here seems unidirectional: from world (i.e., candles) to me. The soothing light of the candles may help shape my mood. But my affective response doesn't affect a material change in the candles-a bidirectional interplay SA proponents argue is necessary for genuine integration with external resources to occur (Sutton 2010). Still, there are many other cases that do involve tight bidirectional engagements, operating both synchronically and diachronically, whereby external resources take over and scaffold regulative dynamics in an ongoing way and, in so doing, open up new forms of experience and expression.

\section{Forms of Affective Scaffolding}

Before turning to psychopathology, I first distinguish three forms of "affective scaffolding": beyond-the-head resources that drive and regulate our affective states. Three forms of scaffolding relevant here are embodied, social, and material scaffolding.

\subsection{Embodied Scaffolding}

No one doubts, of course, that brains are necessary for the occurrence of affective states. But multiple strands of empirical work suggest that brains alone are not sufficient for affective states. The latter are scaffolded not just by neural processes but also by their integration with physical resources spatially distributed throughout the extra-neural body.

Colombetti (2017) has recently argued that an explanation of the physical basis of mood disorders, which involve changes in neurotransmitters and neuromodulators, must involve more than just brain activity. Neurotransmitters and neuromodulators are not sealed off from the body but instead interact with other parts of the organism. And these interactions involve an array of extra-neural processes operating at multiple time-scales: e.g., fluctuating rates of blood glucose in the bloodstream; hormonal processes (released by endocrine glands in the brain and body); complex chemical activities synthesized locally in different areas of the body during stress responses; mood- and behavior-influencing gut microbiota, etc. The takeaway point is that the essential role these processes play in generating moods strongly suggests the latter's physical basis consists of integrated processes looping through the brain and extra-neural body. 
Other work extends affectivity beyond our neurophysiology to the outer limits of our embodiment. There is evidence that some emotions may be scaffolded by behavioral expressions like facial expressions, gestures, and patterns of interpersonal entrainment. One line of evidence comes from deficit studies: cases where an emotion's bodily expression is compromised or missing, such as a form of congenital bilateral facial paralysis called Moebius Syndrome (Cole and Spalding 2009). Some people with Moebius Syndrome report phenomenologically diminished emotional lives (e.g., "I sort of think happy of think sad, not really saying or recognizing actually feeling happy or feeling sad") and struggle regulating their emotions (Krueger and Henriksen 2016). A similar effect is observed in individuals who receive Botox injections inhibiting facial expressions (Baumeister et al. 2016), or in cases of acquired facial paralysis such as Bell's Palsy (Cole 1998). Other work suggests that individuals who suffer severe spinal cord injuries report less-intense feelings of emotions like fear, anger, or sexual arousal (Chwalisz et al. 1988).

For our purposes, there are two important takeaway points. First, multiple lines of evidence suggest that some affective experiences are scaffolded by a range of physical processes distributed throughout and to the edges of our body. Second, some regulative processes likewise loop through this affective scaffolding and modulate the character of our experience.

This latter point can be sharpened by observing that how an affective experience feels is often a function of how it's regulated. And the latter is a process invoking features of our extra-neural body such as agency, expression, and attention. Individuals can up-regulate anger or pain experience simply by frowning, for example (Marzoli et al. 2013); conversely, they can dampen the experience and shorten its temporal duration by inhibiting its physical or vocal expression (Duclos and Laird 2001). Manipulating emotion-specific expressions like smiling or frowning even modulates how we process the same stimulus, such as the perceived funniness of a cartoon or the friendliness of another person (Ohira and Kurono 1993).

As we negotiate different spaces in everyday life (work, home, school, etc.), we adopt a range of flexible, contextsensitive bodily strategies for regulating affect. We adopt a more reserved and less expressive countenance at work or a formal setting than we do at home or when out with friends. But these bodily strategies and the manner by which we enact them nevertheless remain unique to us; they are part of our affective style: our habitual way of moving, acting, expressing, and regulating affect (Colombetti 2016). For most of us, this affective style is largely enacted spontaneously, without deliberate thought. Our body and its expressive resources are experientially present as scaffolding immediately available to us in crafting our distinctive affective style on a moment-to-moment basis. As we'll see shortly, this is not the case with some schizophrenic patients. Many of them are experientially alienated from their bodies and thus lack reliable access to this embodied scaffolding, along with the regulative strategies it supports.

\subsection{Social Scaffolding}

A second way to motivate SA is by considering social scaffolding present both in our dyadic engagements as well in more complex processes operating at group and sociocultural levels. An exhaustive analysis is beyond present concerns (see Parkinson et al. 2005). A few examples will suffice.

Consider first social scaffolding in early infancy. Because they lack endogenous mechanisms for attentional control and affect regulation, young infants depend upon ongoing input from caregivers to realize these capacities. This input is primarily provided by social scaffolding comprised of direct bodily contact and vocal exchanges. For example, much research has shown that by using different strategies to regulate infant attention-smiling, vocal modifications, singing, caressing, diverting or guiding attention away from objects of distress-infants and caretakers tightly integrate their expressive displays and realize affective convergence (Krueger 2013b). This is an early instance of a co-regulatory process distributed across social scaffolding encompassing both infant and caretaker (Taipale 2016).

Social scaffolding remains present throughout our life. When we interact with others, their expressive actionsgestures, facial expressions, postural adjustments, intonation patterns, movements and manipulations of shared space, etc.- - directly impact our bodily responses. These mutuallymodulatory expressions comprise the dynamics of "wespace", or the shared space of our face-to-face engagements (Krueger 2011). A smile and friendly gesture, for instance, elicits similar responses from me and motivates an array of further friendly responses; a threatening gesture or aggressive movement, conversely, compels me to tense up and prepare for my own aggressive response. My embodied scaffolding is in this way dynamically integrated or "coupled" with that of others (De Jaegher and Di Paolo 2007). The kinetics, intensity, and timing of their affective expressions shape my ongoing responses, which in turn feeds back onto and shape their responses, which return to me and impact my ongoing responses, etc.

This socially distributed feedback loop regulates the affective dynamics of group engagements, too. When I am drawn into the exuberance of a lively party, say, or swept along by the collective rage of a political protest or the euphoria of a live concert with thousands of people, the expressions of others literally take hold of my body and pull responsive movements and affective responses out of me 
that diachronically integrate with those of the crowd (Chartrand and Bargh 1999; Slaby 2014). This process "creates a circular interplay of expressions and reactions running in split seconds and constantly modifying each partner's bodily state, in a process that becomes highly autonomous and is not directly controlled by the partners" (Froese and Fuchs 2012 , p. 213). In other words, I am absorbed by a socially distributed co-regulatory system partially comprised of scaffolding beyond my head.

Many bodily processes that make up this scaffolding, such as motor mimicry and movement synchrony, are involuntary. The former refers to spontaneous mimicry of others' facial expressions, gestures, movements, and intonation patterns; the latter, synchronizing speech rhythms and bodily movements over short and long-term timescales (Bernieri and Rosenthal 1991; Wiltermuth and Heath 2009). A core affective function of this scaffolding is the promotion of social cohesion. When present, it enhances feelings of connectedness, rapport, and cooperation (Lakin and Chartrand 2003; van Baaren et al. 2004).

Although this scaffolding is largely constructed on an involuntary ad hoc basis, individuals do have some control over the way they access and exploit it. Knowing how and when to do this is a feature of effective self-maintenance and social functioning - another dimension of an individual's affective style. For example, there is evidence that recovery from negative emotional episodes is augmented by selectively attuning to smiling faces (Koole and Jostmann 2004), particularly in individuals who exhibit strong emotional resilience and self-esteem (DeWall et al. 2011). Presumably, this attunement triggers mimetic responses that up-regulate the individual's negative affective state. It thus seems that strategic attunement to and use of social scaffolding in wespace is crucial for successful affect regulation and a way to cope with an ever-changing social world.

\subsection{Material Scaffolding}

A third way to motivate SA is by looking at the integration of affectivity with material culture: things, spaces, and places comprising our everyday environments. Some argue that making music is an instance affective scaffolding (Cochrane 2008; Geeves and Sutton 2015; Roberts 2015). When musicians practice with their instrument, this engagement is an ongoing process of affective self-stimulation: physical qualities of the instrument and auditory properties of the produced sound coax affective responses out of the performer and synchronically regulate the character of their experience. When actively integrated with their instrument this way, musicians realize affective states with a particular intensity, complexity, and temporal character only possible when part of this materially-scaffolded musical feedback loop.
A similar integration-cum-regulation can occur in music listening, too (Krueger 2014a). Listeners routinely use technologies like MP3 players, smartphones, and music streaming services to scaffold affect (Skånland 2013). These technologies are both portable and highly personalized: we manipulate self-selected playlists specifically to elicit different emotional motor responses. And these manipulations loop back onto us, shaping our future manipulations and responsive behavior. Auditory properties of the music directly modulate a cascade of affect-driving neural and physiological responses, coaxing experiences out of us and regulating their embodied dynamics in real-time over short and long-term listening episodes (Elvers 2016; Witek et al. 2014). When integrated with musical technologies this way, musical-affective feedback loops scaffold access to experiences and regulatory capacities that might otherwise be inaccessible- even from the earliest stages of life (Krueger 2013a).

There are many other cases where regulatory processes loop through our ongoing interactions with material culture. For example, wearing appropriate hiking shoes while walking down a steep path covered in loose gravel-in contrast to walking the same path in light tennis shoes with a flat sole-directly scaffolds our affective state (Colombetti 2016). We're not directly aware of the shoes themselves in this context, as explicit objects of experience. We focus instead on the path and our interactions with it. But our shoes are nevertheless experientially present as scaffolding through which we experience the path; they are transparently integrated into the suite of sensorimotor skills we use to negotiate this environment. And the ongoing feedback we receive from our shoes regulates how we feel and how the world shows up for us via this feeling (i.e., as affording confident walking vs. a halting or nervous descent). ${ }^{3}$

We can appeal to still other cases, too: from our interactions with items of clothing and accessories like handbags (Kaufmann 2011) or religious artifacts (Krueger 2016), to the "affective atmospheres" of corporate spaces and the people and things that make up these spaces (Slaby 2016). Defenders of SA argue that these instances of materiallyscaffolded affect regulation highlight the decentered nature of many affective experiences - that is, the extent to which the individual's endogenous resources, when working together with some external resource or set of resources, no longer constitutes the sole locus of regulative control.

The important point is that enjoying reliable access to the things and spaces of our material culture-being able to actively integrate with them in an immediate and spontaneous way-is crucial for our ability to stabilize and regulate our affective life. And taken together, then, this survey of

\footnotetext{
${ }^{3}$ Maiese (2015) refers to this phenomenon as "affective framing".
} 
embodied, social, and material scaffolding supports the idea that many affective and self-regulative process are driven and organized, at multiple timescales, by external resources beyond brain and body. Individuals who lack consistent and reliable access to this affective scaffolding - whether embodied, social, or material-lack access to the functional and experiential benefits these external resources provide. The experience of "unworlding" in schizophrenia offers a powerful case study of what happens when individuals become detached from these different forms of affective scaffolding-an experiential disturbance of the scaffolded self.

\section{Schizophrenia as a Self-Disorder}

A common way to characterize schizophrenia is to see positive and negative symptoms as separate entities. The former includes clinical manifestations of hallucinations, delusions, and the like; the latter, a diminishment or loss of normal function (alogia, anhedonia, apathy, etc.). However, phenomenological approaches to psychopathology downplay or even reject this distinction and instead approach patients' experience as an integrated whole (Ratcliffe 2008). They treat schizophrenia as a qualitative transformation of the entire structure of experience, rooted in a disturbance of the minimal or core sense of self (Sass and Parnas 2003; see also Parnas and Henriksen 2016).

According to the so-called "ipseity-disturbance model" (IDM),${ }^{4}$ this core self is a pre-reflective form of self-awareness: the enduring feeling of being a subject for whom conscious states are given in a first-personal mode of presentation. It is said to be a necessary structural feature of experience, inextricable from "the distinct manner, or how, of experiencing" (Zahavi 2014, p. 22). A disturbance of this core self typically manifests in patient reports suggesting that this first-person immediacy of their experience is somehow attenuated: "My I-feeling is diminished"; "My I is disappearing for me" (Parnas and Handest 2003, p. 125). "I am disconnected, disintegrated, diminished...I feel that my real self has left me..." (Kean 2009, p. 1034). Within the framework of IDM, this disturbance is termed diminished self-affection.

Diminished self-affection is often accompanied by a compensatory hyperreflective form of self-monitoring (Fuchs 2010b). Within this stance, habitual aspects of the self normally in the background of awareness-including one's affective style, or the distinctive way one thinks, listens, speaks, expresses emotions, and interacts with others and the material environment - are thrust to the foreground and become objects of intense scrutiny. As a result,

$\overline{4}$ Ipseity is Latin for "self" or "itself". the spontaneity and naturalness of everyday interactions is lost, and individuals struggle to smoothly connect with the world and others. For example, patients report that thinking becomes difficult because their thoughts take on spatial or object-like qualities (Parnas and Handest 2003). Moreover, normally spontaneous actions like gesturing, falling asleep, putting a book on the shelf, or making small talk become impossible to carry out due to their excessive focus on every aspect of their performance (Fuchs 2010a). For IDM, this interplay between diminished self-affection and hyperreflexivity is fundamental to the dynamics of the self-disorder distinctive of schizophrenic experience.

IDM offers rich descriptions that greatly enhance our understanding of what it's like to live with schizophrenia from the first-person perspective. However, by focusing so heavily on anomalies of perception and cognition, there is a risk of downplaying or overlooking the affective and emotional distress at the heart of schizophrenia. ${ }^{5}$ SA can help with this concern. Additionally, by presupposing such a thin conception of the self-again, ipseity conceived of as a minimal form of self-consciousness at the heart of individual conscious states-there is a related risk of overlooking the richly situated character of self-disturbances within SZ, that is, the extent to which aspects of these disturbances are distributed across and modulated by the dynamics of the individual's embodied relationship with others and their material environment. Once more, SA can help deal with this worry and illuminate various dimensions of the disturbed scaffolded self. ${ }^{6}$

\section{5 "Unworlding" as a Disturbance of the Scaffolded Self}

Drawing on Heidegger, Sass (1990) characterizes schizophrenia as a kind of "unworlding". This idea is meant to capture the way that people with schizophrenia can become

\footnotetext{
${ }^{5}$ Hamm et al. (2015) develop a similar concern.

${ }^{6}$ Fuchs (2015) and Ratcliffe (2017) both develop compelling characterizations of schizophrenia that anticipate some things I say here. However, my discussion of SA differs in several respects. First, both focus primarily on disturbances at the interpersonal level. This focus is consistent with my characterization of "social scaffolding". But neither says much about how the individual's disturbed relation with the material environment shapes the character of their affective disorder and regulative dysfunction. Second, both focus on disturbances of intentionality: for Ratcliffe, a breakdown of the modal or anticipatory structure of intentionality, which leads to changes in the structure of interpersonal experience; for Fuchs, a breakdown of motor intentional processes that support our intersubjective capacities. While this focus is again consistent with SA, the latter, as we will see, illuminates additional regulative mechanisms (i.e., forms of "scaffolding") that don't necessarily fall within the purview of intentionality-based accounts.
} 
experientially unmoored from the lived spaces of their everyday environments. Within this unworlding experience, people and things are no longer encountered as "readyto-hand"- as affording a range of immediately perceived interactive possibilities (the way a friendly smile affords conversation or a chair sitting) specified by the norms and conventions tacitly governing the context in which they're encountered. Instead, everyday encounters and projects are experienced as puzzling or devoid of meaning. The world as a whole in this way loses its pragmatic and emotive qualities, and the schizophrenic individual is unworlded to the extent that they lose reliable access to the people and things around them.

For IDM, unworlding can be traced back to a more fundamental ipseity disturbance that disrupts how individuals experientially relate to themselves and the world generally. But in this search for a single trouble générateur - and by ultimately locating this feature inside the head of the individual - the embodied and situated nature of unworlding, along with its affective character, is at risk of being downplayed or overlooked, and the causal complexity of the disorder over-simplified. $^{7}$

I suggest instead that unworlding can be seen not simply as a disturbance of ipseity but of the scaffolded self. More specifically, the distinctive affective character of unworlding partially stems, I propose, from an inability to reliably access and exploit the regulative resources offered by different forms of beyond-the-head affective scaffolding (i.e., embodied, social, and material). Without reliable access to this scaffolding, individuals lose not only their feeling of selfintimacy but also their more pervasive feeling of being persistently embedded in the world. Crucially, with this disturbance of affective scaffolding, they also lose reliable access to the functional and experiential benefits this scaffolding affords, which helps explain particular phenomenological features of their affective disturbance. If this characterization of unworlding is on the right track, it thus challenges the assumption that the affectively-salient mechanisms of unworlding — and perhaps many affective disorders in psychopathology more generally — can be explained by appealing exclusively to internal features of the individual, whether ipseity disturbances or disordered neural mechanisms.

\footnotetext{
7 To be fair, in recent work, Sass $(2007,2014)$ has revised his earlier characterization of schizophrenia as lacking affect and now acknowledges that ipseity disturbances involve an affective dimension. However, for Sass, ipseity disturbances nevertheless remain the generative disorder from which affective disturbances subsequently arise. SA instead sees self- and affective disorders as interrelated and co-arising. SA also offers additional descriptive resources for capturing the complexity and multidimensional character of these affective disturbances, including features (e.g., their regulative dynamics) that don't neatly into IDM frameworks.
}

\subsection{Disturbances of Embodied and Social Scaffolding}

Consider first the way unworlding involves disturbances of both embodied and social scaffolding. These two disturbances should be seen as interrelated, I suggest, because bodies are always bound up in interactive relationships with other bodies defined by patterns of mutual modulation and expressive reciprocity. Disruptions of embodied scaffolding are thus coextensive with disruptions of social scaffolding.

One of the most well-replicated findings in the literature is that people with schizophrenia exhibit less facial and vocal emotional expressiveness than do people without schizophrenia (Kring and Elis 2013). One reason for this deficit is likely due not just to a neurophysiological abnormality (cf. Trémeau 2006) but additionally to the experiential distance many people with schizophrenia feel from their body as a lived through subject of experience and action (Krueger and Henriksen 2016). Persistent experiences of bodily alienation, or loss of embodied self-intimacy, are common in schizophrenia spectrum disorders, although the character and articulation of this experience may vary: "it's like I' $m$ not in my body or not attached to it. It's like my body is an appendix that hangs below me" (Henriksen and Nordgaard 2016, p. 268). Often, the body is experienced as taking on a hyper-objectified or quasi-mechanical character: "I'm blessed with a bladder-emptier that I can turn on and off, and an anal expeller" (Angyal 1936); "I walk like a machine... When I am walking, I look at my legs which are moving forward; I fear to fall by not moving them correctly" (Parnas 2003, p. 227).

As these reports suggest-and as IDM emphasizesunworlding experiences clearly involve a disturbance of the self. However, what's sometimes overlooked in these IDM discussions is that the particular bodily character of this self-disturbance means that patients with schizophrenia lose the ability to spontaneously access the affectively salient self-regulative resources offered by their embodied scaffolding. Because they no longer experientially inhabit their body with the immediate self-intimacy most of us take for granted, the suite of expressive resources normally available - again, bodily and agential components of an individual's affective style like their distinctive gestural patterns, postures, facial expressions, and vocalizations-are inaccessible. And in light of the empirical evidence surveyed above, diminished access to regulative resources in bodily scaffolding would seemingly lead to diminished affective experience more generally.

This seems to be the case with some patients. For example, we find reports like: "I feel the emotions but in a very attenuated way. Sometimes I have the feeling that I have to amplify a certain emotion. I try to hold on to it, grab it before it gets away" (Vodušek et al. 2014, p. 256); "It's like an 
internal block, a block of feelings" (Stanghellini et al. 2014, p. 278). And there is also evidence that people with schizophrenia have more general difficulty adaptively regulating their emotions, too (Horan et al. 2013). Bleuler (1950), for example, describes patients jumping from one emotion to the next, from intense agitation to an exaggeratedly happy mood, only to become tearful and sad a few minutes later.

But a closer look reveals a puzzle. As already noted, much evidence suggests that people with schizophrenia are less expressive than people without schizophrenia. Yet, while some predictably report diminished affective experience, others do not-and some even report more intense affective experiences (along with difficulty regulating these experiences) than those without schizophrenia, despite their diminished bodily expressivity (Kring and Moran 2008). How should we account for this variability?

An internalist strategy is to explain these regulative difficulties in terms of top-down mechanisms, e.g., a disturbance of cognitive control and associated prefrontal cortical circuitry (Horan et al. 2013). But this is an excessively narrow perspective that, once more, overlooks the extent to which regulative processes are comprised of embodied scaffolding dynamically intertwined with other expressive bodies-what I've termed social scaffolding. In light of their diminished bodily expressiveness, people with schizophrenia are not robustly integrated into shared social spaces of faceto-face interactions - we-space-and thus lack consistent, robust access to the functional and experiential benefits that come from integrating with this scaffolding (Henriksen and Nilsson 2017). The unpredictability and disorganization of their affective life reflects this lack of consistent integration.

In support of this claim, there is evidence that schizophrenic patients lack bodily empathy, or the ability to detect and respond to others' facial and gestural expressions (Amminger et al. 2012; Edwards et al. 2002). This is consistent with a more general deficit people with schizophrenia show in terms of processing faces and whole-body emotional expressions (Chan et al. 2010; Van den Stock et al. 2011). These disturbances reflect well-documented difficulties with "perceptual organization" in schizophrenia, including a fragmentation of space and object perception (Silverstein et al. 2017; Uhlhaas and Mishara 2007). These disruption manifests via various phenomenological anomalies: e.g., figure-ground confusion, "visual echoes", loss of perceptual stability, etc.

In the social realm-and to return to the idea of social scaffolding - this disruption means that individuals fail to perceive and respond to social affordances in others: ongoing interactive possibilities presented via their bodily expressions, movements, and vocalizations (i.e., social scaffolding). In other words, others are often not seen as expressive unities animated by thoughts, intentions, and emotions, and as inviting an array of interactive responses. Instead, they are encountered as fragmented constellations of unrelated bits. For example, Sass (1992) quotes Renee, who reports that she "saw the individual features of [a woman's] face, separated from each other: the teeth, then the nose, then the cheeks, then one eye and the other" (p. 50). Another says that "[p]eople turn weirdly about, they make gestures, movements without sense; they are phantoms whirling on an infinite plain, crushed by pitiless electric light" (Sechehaye 1970, p. 44).

This lack of access and responsiveness to others' bodily expressions contributes to the individual's loss of social attunement and feeling of alienation. As Stanghellini observes, the "capacity to get involved in and directly perceive others' mental life is the basic requisite for the familiar feeling with the environment, at home, and in everyday social situations" (Stanghellini 2001, p. 207). An important aspect of this loss of social attunement is bodily empathy (or lack thereof) - mimicry and synchronization of others' gestures, expressions, and intentional actions. Without the ability to enact bodily empathy, however-which normally leads to regulative integration with others-people with schizophrenia instead experience a regulative desynchronization in relation to other people: multi-level disturbances of the "timing" of face-to-face interactions (aspects like gestural synchronization and rhythm) that feed into and reinforce their sense of social disconnectedness and animate their affective disorganization (Van Duppen 2017). As a result, they lack immediate access to the regulative resources normally available in beyond-the-head social scaffolding (recall the earlier discussion of how recovery from negative affect is scaffolded by selectively attuning to and mimicking smiling faces). Without reliable perceptual access to this dyadic scaffolding and its functional benefits, it's not surprising, then, that people with schizophrenia experience unpredictable and disorganized affect. The ability to skillfully inhabit wespace and establish dyadic episodes of socially distributed emotion regulation is a core aspects of our ability to manage the changing dynamics of our day-to-day affective life (Varga and Krueger 2013).

In addition to functional disturbances of face-to-face bodily dynamics, schizophrenic patients also show a general lack of social understanding writ large. In other words, they lack an implicit understanding of the "rules of the game" governing social encounters, a sense of proportion for what is appropriate or relevant in social contexts such as what to say, how to act, how to express and manage 
emotions, etc. They say things like, "I don't really grasp what others are up to...I constantly observe myself while I am together with people, trying to find out what I should say or do. It's easier when I am alone or watching TV" (Fuchs 2015 , p. 200). ${ }^{8}$ The schizophrenic individual thus feels alienated not simply from dyadic encounters but also from everyday social environments because the bodily, social, and material resources that would normally provide regulative scaffolding (e.g., other people, social norms and conventions, material culture, etc.) are experientially unavailable. Without access to these external resources, patients thus experience an intersubjective unworlding that further animates their autistic withdrawal and contributes to the regulative difficulties they experience with respect to their affective style. In the next section, I consider how an experiential alienation from the things and spaces of material culture specifically factor into these affective disturbances.

\subsection{Disturbances of Material Scaffolding}

Disturbances of self-experience and intersubjectivity are relatively well-documented in the literature on schizophrenia, including IDM approaches (Henriksen and Nilsson 2017; Pienkos 2015). The notion of the scaffolded self, I've suggested, can usefully supplement these existing discussions by emphasizing both the richly embodied and interpersonally situated nature of the disorder, that is, the extent to which affective features of schizophrenic unworlding are directly modulated by the individual's disturbed relation with their own bodily scaffolding as well as that of others. But conceiving of schizophrenia as a disturbance of the scaffolded self can also make another useful contribution to existing discussions. It can highlight a central aspect of unworlding experience that has not received much attention thus far: the affective significance of the individual's disturbed relationship with the material environment.

An important takeaway from our earlier consideration of material scaffolding is that it plays a central role in bringing regulative stability and predictability to our everyday affective life. Enjoying reliable access to the things and spaces of our material culture-being able to actively integrate with them in an immediate and spontaneous way-is crucial for our ability to skillfully manage our affective experiences on both a moment-to-moment as well as a long-term basis. This is because these external spaces and structures, when arranged in particular configurations, take over much of the regulative work for us. In other words, we individualize our affective "niches" (at home, work, etc.) and, via this

\footnotetext{
$\overline{8}$ Blankenburg (2001) describes this phenomenon as a "loss of common sense" and argues that it's at the core of schizophrenic autism.
}

individualization, settle into and become entrenched within them-and thus allow ourselves to delegate part of the regulative process to our local material scaffolding while we focus on other things (Colombetti and Krueger 2015).

Everyday life reveals many examples of affective individualization and entrenchment. I've intentionally manipulated the material space of my office, for example, in order to evoke and sustain a calm and welcoming atmosphere the moment that I or others enter into it. I've chosen specific artworks to adorn the walls, placed plants strategically around the room, perpetually stream calming ambient music, maintain a particular level of soft lighting, and even arrange the furniture and bookcases to create the regulative space in which this kind of affective experience is predictably elicited and sustained without my explicit attention or effort. We routinely individualize our affective niches in other ways, too, via manipulative strategies that highlight how self-selected items of material culture act directly on aspects of our affective states, modulating their character and function-including the bodily processes (e.g., changes in autonomic nervous system activity, behavioral expressions, etc.) states of action readiness (dispositions to act in certain ways), evaluations or appraisals, and phenomenal experiences that make up the complex architecture of our affective states (Scherer 2009).

For example, we may select brightly colored clothing to counteract the relentless grey of a grim English winter, or a dark-colored "power suit" for a confidence boost before an important job interview. There is evidence that color affects moods (Valdez and Mehrabian 1994), and that the tactile qualities of clothing (e.g., the fuzziness of a familiar sweater) may contribute to the release of chemicals that lower stress levels. Relatedly, the sociologist Jean-Claude Kaufmann (2011) has chronicled the affectively-salient decisions that go into choosing a handbag's contents. For Kaufmann, a handbook isn't merely a utilitarian receptacle for carrying things. It also functions as affective scaffolding. It contains a self-styled collection of technologies chosen specifically to regulate short and long-term affective experiences: good luck charms and tokens for peace of mind (scaffolding one's ability to appraise and cope with difficult situations); snippets of personal notes or letters from loved ones that scaffold fond memories and feelings of nostalgia and connectedness; and small weapons or tools that scaffold feelings of confidence and security, and regulate awareness of relevant action possibilities (e.g., in the face of danger).

To return to an earlier theme: when seamlessly integrated with this material-affective scaffolding, our affective style-including the way we comport ourselves to the environment generally-may differ dramatically than when we lack access to it. If I forget my handbag at home, for example, I may feel more anxious than usual without my good luck charms, or exhibit uncharacteristic timidity whilst navigating the city at night knowing that I lack 
ready access to the mace spray normally nestled in the bottom of that handbag. Similarly, when I am in my office and intensely focused on my writing, the music streaming in the background reliably scaffolds my attention and affect. If it suddenly stops, however-if there is network trouble that interferes with my internet connection-I will be jolted out of this writing-directed entrenchment and forced to recalibrate my affective experience and self-regulative strategies through explicit attention and effort. In other words, without the ongoing regulative input of this integrated network of material scaffolding, my affective style will be dramatically altered; I will need to reorient myself to an affective space suddenly deprived of the background resources I normally trust will be there, working as expected (even when I'm not explicitly focused on them).

These examples highlight how individualization (i.e., crafting our material niches to modulate our particular affective needs) and entrenchment (i.e., settling into and skillfully coping with these self-styled niches) together generate what we might refer to as an implicit affective trust in our environment. When skillfully inhabiting my affective niche, I implicitly trust that it will reliably set up, drive, and regulate my affective experiences in the ways I intend it to (hence my surprise and momentary disorientation when something goes wrong). However, this affective trust in relation to the material environment is precisely what's missing in unworlding experience. This is because the scaffolding used to individualize and become entrenched within our environments is no longer experienced as affording these regulative practices-much the way social scaffolding is not experienced as affording engagement - and a crucial mechanism (or set of mechanisms) for affect regulation unavailable.

As noted previously, schizophrenic patients often exhibit difficulties with perceptual organization. With respect to the material environment, they say things like "Everything is in bits. You put the picture up bit by bit in your head. It's like a photograph that's torn in bits and put together again...If I move there's a new picture that I have to put together again" (McGhie and Chapman 1961, p. 106). Elyn Saks describes a similar perceptual fragmentation when she says that, "No core holds things together...sights, sounds, thoughts, and feelings don't go together. No organizing principle takes successive movements in time and puts them together in a coherent way from which sense can be made" (Saks 2007, p. 13).

A consequence of this perceptual disorganization is that schizophrenic individuals lose their practical grip on the things around them. The world is no longer experienced as presenting an interrelated network of things with determinate meanings and interactive possibilities. Instead, things are saturated with a pervasive sense of strangeness and unfamiliarity-and interactive possibilities diminished or even lost altogether. This loss of material affordances can be seen in the following quotes:

"When, for example, I looked at a chair or a jug, I thought not of their use or function-a jug not as something to hold water and milk, a chair not as something to sit in-but as having lost their names, their functions, their meanings..." (Sechehaye 1970, pp. 55-56).

"Everything around me is immobile. Things appear isolated, each one in itself, without suggesting anything. Certain things which ought to evoke memory, evoke an immense number of thoughts...remain isolated. They are more understood than experienced" (Minkowski 1970, p. 276).

From the perspective of IDM, this loss of practical access to the world can, once again, be traced back to a disruption of the core self as an orienting zero point of consciousness. When this core self is disturbed, the subject loses "the lived point of orientation and the correlated pattern of meanings that make for a coherent and significant world"; in the absence of such a stable first-person perspective, "the structured nature of the worlds of both thought and perception will be altered or even dissolved" (Sass and Parnas 2003, p. 436).

This seems right, as far as it goes. But it's also arguably an incomplete description of the phenomenon. For what is lost here is not simply reliable access to the world's practical significance but, crucially, its affective and regulative significance, too. Within unworlding experience, things and spaces no longer afford self-regulative possibilities, or manifest perceptually as reliable scaffolding one can trust to elicit and regulate predictable affective responses - and one's entire affective orientation to the environment (i.e., one's affective style) is modulated accordingly. Put otherwise, without reliable access to the material environment, possibilities for individualization and entrenchment are lost, leading to a breakdown of affective trust and a disorganization of an individual's affective style.

The distinctive character of this affective disturbance can be understood by looking at case vignettes like the following:

"A 32-year old patient reports that at the age of 16 , he had become more and more uncertain about whether his personal things really belonged to him or had somehow been exchanged by others. When buying books, he was not sure if the salesman had not secretly replaced the ones he had chosen; so he had to give them away and always buy new ones. When leaving things on his school-desk inattentively, he later began to doubt whether they were still the same, 
and had to throw them away. More and more he lost the trust in his environment" (Fuchs 2005, p. 101).

Part of this patient's distrust has to do with ownership of his belongings (i.e., the delusional fear that someone is replacing his belongings with duplicates). But again, there is also critical_and potentially overlooked-affective dimension to this distrust, directly tied to the stability (or lack therefore) of the patient's available material scaffolding. Plagued by the fear that the items in his environment are continually being replaced, the patient no longer can individualize and become comfortably entrenched within a selfstyled network of affective scaffolding. Instead of inhabiting spaces set up to reliably elicit and regulate predictable affective experiences, he must instead devote excessive attention to hyper-reflectively scrutinizing his surroundings, hunting for indications that someone is deliberately manipulating his affective niche. This hyper-reflective stance hinders possibilities for skillful entrenchment. Accordingly, the world and things in it no longer afford smooth self-regulative possibilities or function as trustworthy "affective stabilizers". The surrounding space becomes unpredictable and foreboding, leading to a disorganized and unstable affective style - and an overall diminishment of regulative function and a profound sense of "diminished agency" (Ratcliffe 2013).

Other patient reports similarly speak to this character of unworlding experience:

"Then I felt that the autumn landscape [was pervaded] with a Second space. It was fine and invisible, hardly detectable. The second Space was dark, empty, frightening" (Sass et al. 2017, p. 20).

"Everything was the same and yet it seemed strange" (ibid. p. 42).

"...more and more, despite my efforts, I lost the feeling of practical things" (Sechehaye 1970, p. 29).

The takeaway lesson from these reports, then, is that unworlding is tied not just to a disturbance of minimal selfhood but also to loss of affective trust in the world-and more specifically, trust in the reliable presence of individualized networks of affective scaffolding that normally organize the lived spaces of our material environments. This scaffolding brings both regulative stability and predictability to our affective life, easily-overlooked features of everyday experience that make entrenchment and affective trust possible in the first place. Without reliable access to this scaffolding, however, our ability to manage our affective experiences on both a moment-to-moment as well as a long-term basis is compromised-leading to a functional disturbance of the materially-scaffolded self.

\section{Conclusion}

I have argued that the notion of "affective scaffolding" can be of use to phenomenological psychopathology. After distinguishing three forms of affective scaffolding-embodied, social, and material-I suggested that these forms of affective scaffolding can be fruitfully mapped onto selfdisturbances in schizophrenia. More precisely, I argued that "unworlding" experiences in schizophrenia can be understood not simply as a disturbance of ipseity or minimal selfconsciousness but, additionally, of the scaffolded self-the affective self established and regulated via its ongoing engagement with people and things. This perspective, I've further suggested, can shed light on the causes and consequences of characteristic challenges schizophrenic patients face when it comes to managing their day-to-day affective life (i.e., their affective style). To be clear, the preceding analysis is not offered to replace IDM. Instead, my intention is to enrich this approach by bringing to the foreground affective dimensions of unworlding sometimes downplayed or overlooked by a narrow focus on disturbances of individual self-consciousness.

Where does this leave us? Reconceptualizing selfdisturbances in schizophrenia as experiential disruptions of the scaffolded self can, I propose, lead to at least two substantive consequences. First, this approach can help to further illuminate the causal complexity of schizophrenic self-disorder-and in particular, the extent to which both extra-neural embodied as well as environmental scaffolding comprise some of the regulatory dynamics responsible for the characteristic affective dysfunction. For some proponents of biological psychiatry, psychiatric disorders are a matter of one or more functional neurological failures. ${ }^{9}$ As a consequence of this assumption, the "fMRI dream of psychiatric imaging", as Sprevak (2011) refers to it, is that taxonomizing, diagnosing, and treating psychiatric disorders-including self-disturbances in schizophrenia-can be done by taxonomizing, diagnosing, and treating underlying neurological failures. However, if SA and the notion of the scaffolded self are on the right track, they suggest that the causal mechanisms responsible for affective disturbances in schizophrenia may involve more than a single localizable failure in a particular brain region — or even, perhaps, a single disturbance of a minimal structural feature of consciousness (i.e., ipseity). Instead, insofar as the affective self is

\footnotetext{
${ }^{9}$ Recent examples of this assumption can be found in characterizations of depression (Castrén 2005; Drevets et al. 2008; Pandya et al. 2012) and anxiety (Wehrenberg and Prinz 2007) as brain disorders. Similarly, Insel and Quirion (2005) begin their general defense of psychiatry as a clinical neuroscience discipline by insisting that "mental disorders be understood and treated as brain disorders" (p. 2221).
} 
regulated and maintained via resources distributed throughout the extra-neural body and perhaps even the surrounding environment, it is possible that the relevant breakdown involves multiple disturbances both inside as well as outside the individual, within their ongoing engagement with the people and things around them. An alternative explanatory framework is thus needed to capture the complex interplay of distributed components and processes responsible for this breakdown. SA can offer theoretical resources for developing such a framework.

Second-and following from the first point-this view may have practical consequences for how we approach intervention and treatment. If there is doubt that schizophrenic disturbances have clear-cut underlying neural causes-but instead arise from a more general disturbance of the larger brain-body-environment system - pharmacological strategies targeting specific brain regions will likely be inadequate on their own. Instead, it will be important to develop robustly embodied and situated strategies designed to recalibrate the individual's entire way of being in the world and relating to themselves and others-strategies targeting their affective style.

As the previous analysis suggested, affective disturbances in schizophrenia - and perhaps many mental disorders more generally-are existential, not exclusively neural, insofar as they are subjectively experiential, situated, and agential (Matthews 2007). What is therefore needed are "bottomup treatment methods" that shift bodily dynamics and feelings, and which allow not only for changes in cognition and patterns of thought but also embodied affect and emotion (Maiese 2015). Therapeutic methods like yoga, music, and dance/movement therapies may be examples of such treatment methods. Admittedly, these strategies are relatively new and require further study and careful implementation. However, as Maiese documents in detail, they have already shown promising success in helping people with schizophrenia learn how to re-inhabit and become more attuned to their bodies, deepen their sense of ownership and agency, feel more at home in their surroundings, and develop expressive channels for exploring and regulating their emotions and sharing these emotions with others (i.e., learning how to re-inhabit we-space) (see Maiese 2015, ch. 6).

To conclude, the point, once again, is not that brainside mechanisms play no role whatsoever in mental illness. Clearly they do. But we are more than our brains. What a scaffolded approach reminds us is that who we are is very much a matter of where we are. The continually-changing network of spaces and places, people and things, that comprise our shared world are often part of us - part of the regulative dynamics that make up the scaffolded self.

Acknowledgements I am grateful for feedback on this material from audiences in Cardiff, Lancaster, Madrid, and Los Angeles. Many thanks also to Giovanna Colombetti and Mads Gram Henriksen for helpful comments on earlier versions of this paper. Finally, I would like to thank the two anonymous reviewers for their careful reading and critical suggestions. Their input greatly improved the main argument.

\section{Compliance with Ethical Standards}

Conflict of interest The author declares no conflict of interest.

Ethical Approval This article does not contain any studies with human participants or animals performed by any of the authors.

Open Access This article is distributed under the terms of the Creative Commons Attribution 4.0 International License (http://creativeco mmons.org/licenses/by/4.0/), which permits unrestricted use, distribution, and reproduction in any medium, provided you give appropriate credit to the original author(s) and the source, provide a link to the Creative Commons license, and indicate if changes were made.

\section{References}

Adams F, Aizawa K (2008) The bounds of cognition. Blackwell, Oxford

Amminger G, Schäfer M, Papageorgiou K et al (2012) Emotion recognition in individuals at clinical high-risk for schizophrenia. Schizophr Bull 38(5):1030-1039

Angyal A (1936) The experience of the body-self in schizophrenia. Archiv Neurol Psychiatry 35:1029-1053

Baumeister J-C, Papa G, Foroni F (2016) Deeper than skin deep: The effect of botulinum toxin-A on emotion processing. Toxicon 118:86-90

Bernieri F, Rosenthal R (1991) Interpersonal coordination: behavior matching and interactional synchrony. In: Feldman R, Rime B (eds) Fundamentals of nonverbal behavior. Cambridge University Press, Cambridge, pp 401-432

Blankenburg W (2001) First steps toward a psychopathology of "common sense. Philos Psychiatry Psychol 8(4):303-315

Bleuler E (1950) Dementia praecox or the group of schizophrenias (Zinkin J, trans.). International Universities Press, Oxford

Carter J, Gordon E, Palermos S (2016) Extended emotion. Philos Psychol 29(2):198-217

Castrén E (2005) Is mood chemistry? Nat Rev Neurosci 6(3):241-246

Chan R, Li H, Cheung E et al (2010) Impaired facial emotion perception in schizophrenia: a meta-analysis. Psychiatry Res 178(2):381-390

Chartrand T, Bargh J (1999) The chameleon effect: the perception-behavior link and social interaction. J Pers Soc Psychol 76(6):893-910

Chwalisz K, Diener E, Gallagher D (1988) Autonomic arousal feedback and emotional experience: evidence from the spinal cord injured. J Pers Soc Psychol 54(5):820-828

Clark A (2008) Supersizing the mind: embodiment, action, and cognitive extension. Oxford University Press, Oxford

Cochrane T (2008) Expression and extended cognition. J Aesthet Art Crit 66(4):329-340

Cole J (1998) About face. MIT Press, Cambridge

Cole J, Spalding H (2009) The invisible smile: living without facial expression. Oxford University Press, Oxford

Colombetti G (2016) Affective incorporation. In: Simmons JA, Hackett JE (eds) Phenomenology for the twenty-first century. Palgrave Macmillan, London, pp 231-248 
Colombetti G (2017) The embodied and situated nature of moods. Philosophical Quarterly of Israel, Philosophia

Colombetti G, Krueger J (2015) Scaffoldings of the affective mind. Philos Psychol 28(8):1157-1176

Colombetti G, Roberts T (2015) Extending the extended mind: the case for extended affectivity. Philos Stud 172(5):1243-1263

Cooper R (2017) Where's the problem? Considering Laing and Esterson's account of schizophrenia, social models of disability, and extended mental disorder. Theor Med Bioeth 38(4):239-244

De Jaegher H, Di Paolo E (2007) Participatory sense-making. Phenomenol Cogn Sci 6(4):485-507

DeWall C, Deckman T, Pond R et al (2011) Belongingness as a core personality trait: how social exclusion influences social functioning and personality expression. J Pers 79(6):1281-1314

Drayson Z (2009) Embodied cognitive science and its implications for psychopathology. Philos Psychiatry Psychol 16(4):329-340

Drevets W, Price J, Furey M (2008) Brain structural and functional abnormalities in mood disorders: implications for neurocircuitry models of depression. Brain Struct Funct 213(1-2):93-118

Duclos S, Laird J (2001) The deliberate control of emotional experience through control of expressions. Cogn Emot 15(1):27-56

Edwards J, Jackson H, Pattison P (2002) Emotion recognition via facial expression and affective prosody in schizophrenia: a methodological review. Clin Psychol Rev 22(6):789-832

Elvers P (2016) Songs for the ego: theorizing musical self-enhancement. Front Psychol 7(2):1-11

Froese T, Fuchs T (2012) The extended body: a case study in the neurophenomenology of social interaction. Phenomenol Cogn Sci 11(2):205-235

Fuchs T (2005) Corporealized and disembodied minds: a phenomenological view of the body in melancholia and schizophrenia. Philos Psychiatry Psychol 12(2):95-107

Fuchs T (2010a) Phenomenology and psychopathology. In: Schmicking D, Gallagher S (eds) Handbook of phenomenology and cognitive science. Springer Netherlands, Dordrecht, pp 546-573

Fuchs T (2010b) The psychopathology of hyperreflexivity. J Specul Philos 24(3):239-255

Fuchs T (2015) Pathologies of intersubjectivity in autism and schizophrenia. J Conscious Stud 22(1-2):191-214

Geeves A, Sutton J (2015) Embodied cognition, perception, and performance in music. Empir Musicol Rev 9(3-4):247-253

Greenwood J (2013) Contingent transcranialism and deep functional cognitive integration: the case of human emotional ontogenesis. Philos Psychol 26(3):420-436

Gross J (2015) Emotion regulation: current status and future prospects. Psychol Inq 26(1):1-26

Hamm J, Buck B, Lysaker P (2015) Reconciling the ipseity-disturbance model with the presence of painful affect in schizophrenia. Philos Psychiatry Psychol 22(3):197-208

Henriksen MG, Nilsson LS (2017) Intersubjectivity and psychopathology in the schizophrenia spectrum: complicated we, compensatory strategies, and self-disorders. Psychopathology 50(5):321-333

Henriksen MG, Nordgaard J (2016) Self-disorders in schizophrenia. In: Stanghellini G, Aragona M (eds) An experiential approach to psychopathology - what is it like to suffer from mental disorder? Springer International Publishing, Switzerland, pp 265-280

Hoffman G (2016) Out of our skulls: how the extended mind thesis can extend psychiatry. Philos Psychol 29(8):1160-1174

Horan W, Hajcak G, Wynn J et al (2013) Impaired emotion regulation in schizophrenia: evidence from event-related potentials. Psychol Med 43(11):2377-2391

Insel T, Quirion R (2005) Psychiatry as a clinical neuroscience discipline. J Am Med Assoc 294(17):2221-2224
Kaufmann J-C (2011) Le sac: un petit monde d'amour. Jean-Claude Lattès, Paris

Kean C (2009) Silencing the self: schizophrenia as a self-disturbance. Schizophr Bull 35(6):1034-1036

Koole S, Jostmann N (2004) Getting a grip on your feelings: effects of action orientation and external demands on intuitive affect regulation. J Pers Soc Psychol 87(6):974-990

Koole S, Veenstra L (2015) Does emotion regulation occur only inside people's deads? Toward a situated cognition analysis of emotionregulatory dynamics. Psychol Inq 26(1):61-68

Kring A, Elis O (2013) Emotion deficits in people with schizophrenia. Annu Rev Clin Psychol 9:409-433

Kring A, Moran E (2008) Emotional response deficits in schizophrenia: insights from affective science. Schizophr Bull 34(5):819-834

Krueger J (2011) Extended cognition and the space of social interaction. Conscious Cogn 20(3):643-657

Krueger J (2013a) Empathy, enaction, and shared musical experience: evidence from infant cognition. In: Cochrane T, Fantini B, Scherer K (eds) The emotional power of music: multidisciplinary perspectives on musical expression, arousal, and social control. Oxford University Press, Oxford, pp 177-196

Krueger J (2013b) Ontogenesis of the socially extended mind. Cogn Syst Res 25-26:40-46

Krueger J (2014a) Affordances and the musically extended mind. Front Psychol 4(1003):1-13

Krueger J (2014b) Varieties of extended emotions. Phenomenol Cogn Sci 13(4):533-555

Krueger J (2016) The extended mind and religious cognition. In: Clements N (ed) Religion: mental religion. Part of the Macmillan interdisciplinary handbooks: religion series. MacMillan, New York, pp 237-254

Krueger J, Henriksen MG (2016) Embodiment and affectivity in moebius syndrome and schizophrenia: a phenomenological analysis. In: Simmons JA, Hackett JE (eds) Phenomenology for the 21st century. Palgrave Macmillan, London, pp 249-267

Krueger J, Szanto T (2016) Extended emotions. Philos Compass 11:863-878

Lakin J, Chartrand T (2003) Using nonconscious behavioral mimicry to create affiliation and rapport. Psychol Sci 14(4):334-339

Maiese M (2015) Embodied selves and divided minds. Oxford University Press, New York

Maiese M (2016) Affective scaffolds, expressive arts, and cognition. Front Psychol 7(359):1-11

Marzoli D, Custodero M, Pagliara A et al (2013) Sun-induced frowning fosters aggressive feelings. Cogn Emot 27(8):1513-1521

Matthews E (2007) Body-subjects and disordered minds: treating the "whole" person in psychiatry. Oxford University Press, Oxford

McGhie A, Chapman J (1961) Disorders of attention and perception in early schizophrenia. Br J Med Psychol 34(2):103-116

Minkowski E (1970) Lived time: phenomenological and psychopathological studies. Northwestern University Press, Evanston

Ohira H, Kurono K (1993) Facial feedback effects on impression formation. Percept Mot Skills 77:1251-1258

Pandya M, Altinay M, Malone D et al (2012) Where in the brain is depression? Curr Psychiatry Rep 14(6):634-642

Parkinson B, Fischer A, Manstead A (2005) Emotions in social relations. Psychology Press, New York

Parnas J (2003) Self and schizophrenia: a phenomenological perspective. In: Kircher T, David A (eds) The self in neuroscience and psychiatry. Cambridge University Press, Cambridge, pp 218-241

Parnas J, Handest P (2003) Phenomenology of anomalous self-experience in early schizophrenia. Compr Psychiatry 44(2):121-134

Parnas J, Henriksen MG (2016) Mysticism and schizophrenia: A phenomenological exploration of the structure of consciousness in the schizophrenia spectrum disorders. Conscious Cogn 43:75-88 
Pienkos E (2015) Intersubjectivity and its role in schizophrenic experience. Humanist Psychol 43(2):194-209

Ratcliffe M (2008) Feelings of being: phenomenology, psychiatry and the sense of reality. Oxford University Press, Oxford

Ratcliffe M (2013) Delusional atmosphere and the sense of unreality. In: Stanghellini G, Fuchs T (eds) One century of Karl Jaspers' general psychopathology. Oxford University Press, Oxford, pp 229-244

Ratcliffe M (2017) Real hallucinations: psychiatric illness, intentionality, and the interpersonal world. MIT Press, Cambridge

Roberts T (2015) Extending emotional consciousness. J Conscious Stud 22(3-4):108-128

Saks E (2007) The center cannot hold: my journey through madness. Hyperion, New York

Sass L (1990) The truth-taking-stare: a Heideggerian interpretation of a schizophrenic world. J Phenomenol Psychol 21(2):121-149

Sass L (1992) Madness and modernism: insanity in the light of modern art, literature, and thought. Basic Books, New York

Sass L (2007) Contradictions of emotion in schizophrenia. Cogn Emot 21(2):351-390

Sass L (2014) Self-disturbance and schizophrenia: structure, specificity, pathogenesis. Schizophr Res 152(1):5-11

Sass L, Parnas J (2003) Schizophrenia, consciousness, and the self. Schizophr Bull 29(3):427-444

Sass L, Pienkos E, Škodlar B et al (2017) EAWE: examination of anomalous world experience. Psychopathology 50(1):10-54

Scherer K (2009) The dynamic architecture of emotion: evidence for the component process model. Cogn Emot 23(7):1307-1351

Sechehaye M (1970) Autobiography of a schizophrenic girl. (RubinRabson G trans). New American Library, New York

Silverstein S, Demmin D, Škodlar B (2017) Space and objects: on the phenomenology and cognitive neuroscience of anomalous perception in schizophrenia. Psychopathology 50(1):60-67

Skånland M (2013) Everyday music listening and affect regulation: the role of MP3 players. Int J Qual Stud Health Well-Being 8(20595): $1-10$

Slaby J (2014) Emotions and the extended mind. In: Salmela M, Von Scheve C (eds) Collective emotions. Oxford University Press, Oxford, pp 32-46

Slaby J (2016) Mind invasion: situated affectivity and the corporate life hack. Front Psychol 7(266):1-13

Sprevak M (2011) Neural sufficiency, reductionism, and cognitive neuropsychiatry. Philos Psychiatry Psychol 18(4):339-344
Stanghellini G (2001) Psychopathology of common sense. Philos Psychiatry Psychol 8(2):201-218

Stanghellini G, Ballerini M, Lysaker P (2014) Autism rating scale. J Psychopathol 20:273-285

Stephan A, Walter S, Wilutzky W (2014) Emotions beyond brain and body. Philos Psychol 27(1):65-81

Sterelny K (2010) Minds: extended or scaffolded? Phenomenol Cogn Sci 9(4):465-481

Sutton J (2010) Exograms and interdisciplinarity: history, the extended mind, and the civilizing process. In: Menary R (ed) The extended mind. MIT Press, Cambridge, pp 189-225

Taipale J (2016) Self-regulation and beyond: affect regulation and the infant-caregiver dyad. Front Psychol 7(889):1-13

Trémeau F (2006) A review of emotion deficits in schizophrenia. Dialogues Clin Neurosci 8(1):59-70

Uhlhaas P, Mishara A (2007) Perceptual anomalies in schizophrenia: integrating phenomenology and cognitive neuroscience. Schizophr Bull 33(1):142-156

Valdez P, Mehrabian A (1994) Effects of color on emotions. J Exp Psychol 123(4):394-409

Van Duppen Z (2017) The intersubjective dimension of schizophrenia. Philosophy, Psychiatry

van Baaren R, Holland R. Kawakami K et al (2004) Mimicry and prosocial Behavior. Psychol Sci 15(1):71-74

Van den Stock J, de Jong S, Hodiamont P et al (2011) Perceiving emotions from bodily expressions and multisensory integration of emotion cues in schizophrenia. Soc Neurosci 6(5-6):537-547

Varga S, Krueger J (2013) Background emotions, proximity and distributed emotion regulation. Rev Philos Psychol 4(2):271-292

Vodušek V, Parnas J, Tomori M et al (2014) The phenomenology of emotion experience in first-episode psychosis. Psychopathology 47(4):252-260

Wehrenberg M, Prinz S (2007) The anxious brain: the neurobiological basis of anxiety disorders and how to effectively treat them. WW Norton \& Company, New York

Wiltermuth S, Heath C (2009) Synchrony and cooperation. Psychol Sci 20(1):1-5

Witek M, Clarke E, Wallentin M et al (2014) Syncopation, bodymovement and pleasure in groove music. PloS ONE 9(4):e94446

Zahavi D (2014) Self and other: exploring subjectivity, empathy, and shame. Oxford University Press, Oxford 